\title{
Estudio del comportamiento de un Control MPC [Control Predictivo Basado en el Modelo] comparado con un Control PID en una Planta de Temperatura
}

\author{
Study of the Performance of a MPC Control [Model Predictive \\ Control] Compared with a PID Control on a Temperature Plant
}

\section{Estudo do comportamento de um Controle MPC [Controle Preditivo Baseado no Modelo] comparado com um Controle PID em uma Usina de Temperatura}

\author{
Emil Hernández-Arroyo* \\ Jorge Luis Díaz-Rodríguez** \\ Omar Pinzón-Ardila***
}

\section{Resumen}

Presenta un estudio comparativo entre el Control Predictivo basado en el Modelo [MPC] y el control PID, en una planta piloto de temperatura. Se encontró que el control MPC presenta mejor comportamiento, con un tiempo de asentamiento de 1000 segundos y una sobre-elongación de $5{ }^{\circ} \mathrm{C}$, y que el PID presenta un tiempo de asentamiento de 2000 segundos y una sobre-elongación de $40{ }^{\circ} \mathrm{C}$. Simultáneamente, se presenta una forma alternativa para controlar y monitorear en tiempo real la variable temperatura; para ello se dispone de un computador de escritorio que utiliza el software MATLAB 7.1 y la herramienta Real-Time Windows Target.

Palabras clave: Control predictivo, Control PID, Control no lineal, Control MPC, Modulación de ancho de pulso, Planta de temperatura.

\footnotetext{
* M.Sc. Universidad Pontificia Bolivariana (Bucaramanga-Santander, Colombia).emil.hernandez@upb.edu.co

** M.Sc. Universidad de Pamplona (Pamplona-Norte de Santander, Colombia).

*** Ph.D. Universidad Pontificia Bolivariana (Bucaramanga-Santander, Colombia).
} 


\section{Abstract}

This project aims to conduct a comparative study of the Model Predictive Control [MPC] and the classical controller PID, over a pilot temperature plant. From the experimental results, was found that MPC has better behavior with a assessment time of 1000 seconds and a over shoot of $5^{\circ} \mathrm{C}$, the assessment time of PID control was 2000 seconds and over shoot of $40{ }^{\circ} \mathrm{C}$. At the same time an alternative way was presented to controlling and monitoring in real time the temperature variable and is available desktop computer using the software tool MATLAB 7.1 and Real-Time Windows Target.

Keywords: Predictive Control, PID Controller, MPC Controller, Nonlinear Control, Pulse Width Modulation (PWM), Temperature Plant.

\section{Resumo}

Apresenta um estudo comparativo entre o Controle Preditivo baseado no Modelo [MPC] e no controle PID, numa usina piloto de temperatura. Encontrou-se que o controle MPC apresenta melhor comportamento, com um tempo de assentamento de 1000 segundos e uma sobreelongação de $5{ }^{\circ} \mathrm{C}$, e que o PID apresenta um tempo de assentamento de 2000 segundos e uma sobreelongação de $40^{\circ} \mathrm{C}$. Simultaneamente, se apresenta uma forma alternativa para controlar e monitorar em tempo real a variável temperatura; para isso se dispõe de um computador que utiliza o software MATLAB 7.1 e a ferramenta Real-Time Windows Target.

Palavras chave: Controle preditivo, Controle PID, Controle não lineal, Controle MPC, Modulação de largura de pulso, Usina de temperatura. 


\section{INTRODUCCIÓN}

El control automático ha desempeñado un papel importante en el avance de la ingeniería y la ciencia; dada su gran importancia en diferentes sistemas, tales como vehículos espaciales y guiado de misiles y robots, el control se ha convertido en parte integral de los procesos modernos industriales y de fabricación. Por ejemplo, el control automático es fundamental para el control de presión, temperatura, humedad, viscosidad, nivel y caudal en las industrias de control de procesos, para mejorar la productividad y simplificar el trabajo de muchas operaciones manuales repetitivas $y$ rutinarias, consiguiendo con esto un comportamiento óptimo de los sistemas [1].

Numerosos estudios han investigado el desempeño de los controladores predictivos basados en el modelo MPC [2-3]. Salcedo y Correa [2] diseñaron un simulador para control predictivo, versión modular, que también permite configurar estrategias de control PID convencionales. Gómez y Correa [3] implementaron un sistema de control predictivo multivariable en un horno y lograron incrementar su eficiencia y la vida útil de sus componentes. En algunos sistemas físicos existe un retardo importante entre la acción y la respuesta del sistema; esto es típico de los sistemas de control de temperatura, debido principalmente a dos razones: la primera es que estos sistemas pueden tener una dinámica lenta propia de los sistemas térmicos; la segunda es debido al retardo de transporte, o tiempo muerto, producido principalmente por el sistema de tuberías que poseen la mayoría de ellos.

Durante mucho tiempo ha sido práctica común el análisis y diseño de sistemas de control lineales para el control de sistemas de temperatura utilizando un controlador de tipo Proporcional, Integral y Derivativo (PID) donde se han obtenido resultados aceptables [4$6]$.

El desempeño de un controlador PID para sistemas de control de temperatura puede mejorarse añadiendo una acción predictiva para compensar el retardo de la respuesta de este tipo de sistema. Aunque la acción derivativa es una alternativa de predicción, existen actualmente otras basadas principalmente en alternativas de control no lineal. El predictor Smith (SP) y el MPC son útiles para sistemas que incluyen tiempos de retardo, y cuando se cuenta con buenas aproximaciones del modelo de la planta, con lo cual se pueden conseguir drásticas mejoras en la respuesta transitoria ante eventuales cambios en la señal de referencia. El controlador PID predictivo es una versión simple del predictor de Smith. El MPC es una generalización del predictor Smith que se puede aplicar satisfactoriamente a sistemas con múltiples entradas y múltiples salidas.

Dado que los controladores predictivos se fundamentan en los modelos matemáticos del sistema a controlar es importante que el modelo sea lo más preciso posible; por lo tanto, es particularmente importante tener una adecuada estimación del retardo que introduce el sistema [5].

Un controlador PI solamente considera datos pasados y presentes, y un controlador PID también predice el comportamiento futuro del proceso por extrapolación lineal; sin embargo, una buena predicción puede mejorar el desempeño de los controladores, particularmente cuando el proceso presenta retardos, lo cual es común en el control de procesos. Los retardos se pueden deber a un mecanismo de retardo puro, causado por el transporte o el tiempo requerido para los cálculos y la comunicación, o a mediciones obtenidas en un análisis off-line; también aparecen cuando un sistema de alto orden o una ecuación diferencial parcial se aproximan a un modelo de bajo orden, como es el ejemplo de la conducción de calor.

Por otro lado, existen controladores que pueden tratar este tipo de procesos. El MPC es una clase amplia de controladores que se está popularizando y es objeto de nuestro estudio [7].

\section{INSTALACIÓN EXPERIMENTAL}

En la Figura 1 se muestra el esquema del proyecto realizado. En este trabajo se presenta una forma alternativa para controlar y monitorear en tiempo real la variable temperatura del equipo fabricado por la empresa Grupo Siatec ltda. Para ello se dispone de un computador de escritorio que utiliza el software MATLAB 7.1 y la herramienta Real-Time Windows Target. Este software es ampliamente extendido, debido a que permite simular, monitorear y controlar las variables del proceso en una misma plataforma 
computacional. Además, se está controlando el módulo mediante algoritmos con arquitectura abierta (sistemas no propietarios), los cuales permiten una mejor adaptabilidad y diseño del algoritmo de control. Lo anterior permite explorar diferentes formas de control sin necesidad de depender de arquitecturas cerradas [8-10]. Este módulo se conecta a un computador de escritorio utilizando una tarjeta de adquisición de datos PCI-6032 E de la empresa National Instruments. Como primer paso, se diseña e implementa la señal de control por modulación de ancho de pulso (PWM), la cual regula los tiempos de encendido de la planta de temperatura, por medio del relé de estado sólido. Con esta señal PWM, implementada en simulink, se puede controlar la variable temperatura; además, se diseñan e implementan los circuitos acondicionadores de señal (CAS) para adquirir la señal del sensor de temperatura del módulo mediante una tarjeta de adquisición de datos (DAQ). Luego se diseñan los reguladores: PID y MPC, mediante los diagramas de bloques de SIMULINK para controlar la variable temperatura. El programa de control en tiempo real se genera utilizando la herramienta Real-Time Windows Target de MATLAB, interactuando físicamente sobre el módulo, permitiendo la posibilidad de hacer un ajuste del control y de los parámetros del regulador para optimizar la respuesta del sistema [11].

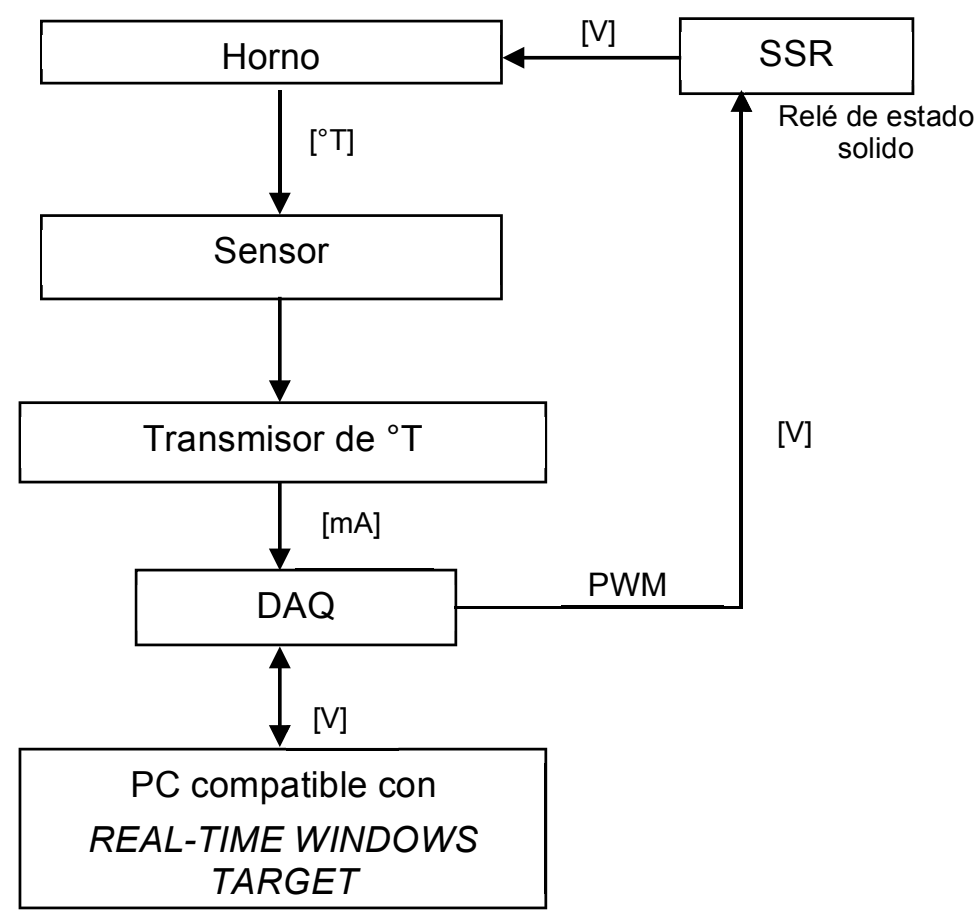

Fig. 1. Diagrama de bloque sistema de control de temperatura

\section{Metodología}

\section{A. Planta piloto de temperatura}

La planta de temperatura (Figura 1) consiste en un horno con resistencias eléctricas, un transmisor de temperatura PT100 SITRANS TKL SIEMENS, una fuente de $24 \mathrm{~V}$ y alimentación de $110 \mathrm{~V}$, donde se estudian los fundamentos del control de procesos, teniendo la temperatura como variable a controlar.

En la planta de temperatura se usó una tarjeta de adquisición de datos (DAQ), que se encarga de efectuar la comunicación entre el computador y el módulo didáctico según se observa en la Figura 2. 


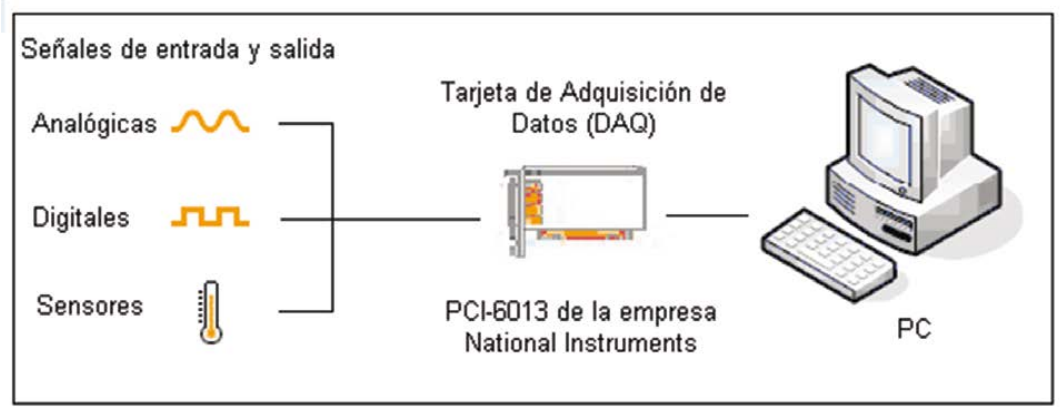

Fig. 2. Sistema de adquisición de datos

\section{B. Obtención del modelo matemático de la planta}

Se determinó el modelo matemático de la planta, realizando un proceso de identificación usando información de la entrada (voltaje de $120 \mathrm{~V}$ ) y adquiriendo la variable temperatura $\left[{ }^{\circ} \mathrm{C}\right]$ con la planta en lazo abierto. En la Figura 3 se observa el diagrama de bloques en simulink que permite obtener el modelo de la planta en lazo abierto.

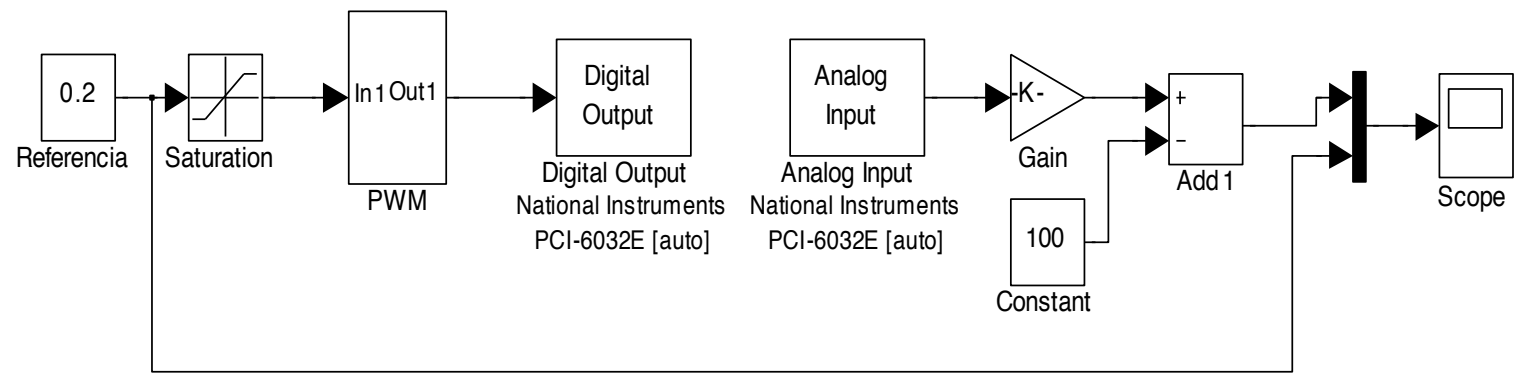

Fig. 3. Diagrama de bloques en lazo abierto para realizar la identificación de la planta

En la Figura 4 se observa el resultado de la identificación con la cual se puede determinar un modelo aproximado de la planta.

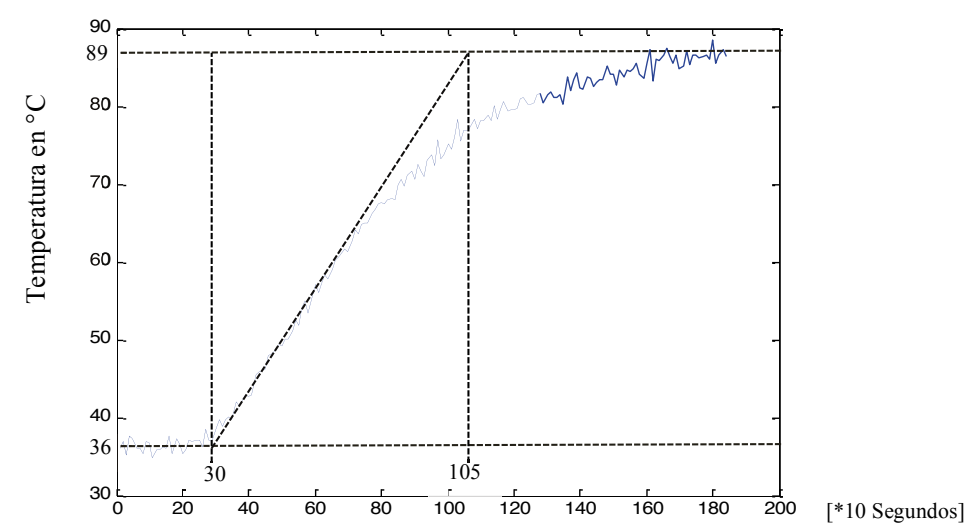

Fig. 4. Respuesta de la planta en lazo abierto 
De acuerdo con la curva de reacción o la repuesta de la planta que se muestra en la Figura 4, se obtiene la constante de tiempo cuando la pendiente se toma desde el origen $\left(36^{\circ} \mathrm{C}\right)$, realizando una aproximación a un sistema de primer orden con retardo por el método de la pendiente máxima [8], y terminado el retardo, a los $300 \mathrm{~s}$, intercepta el valor máximo de la respuesta $\left(89^{\circ} \mathrm{C}\right)$, en este caso, en $1050 \mathrm{~s}$, luego la constante de tiempo se determina como $1050-300=750 \mathrm{~s}$. La función de transferencia de la planta queda expresada según la ecuación(1).

$$
G p(s)=\frac{k e^{-L s}}{1+T s}=\frac{0.44 e^{-300 s}}{1+750 s}
$$

Donde:

$\mathrm{L}=300 \mathrm{~s}$ (Tiempo de retardo)

$\mathrm{T}=750 \mathrm{~s}$ (Constante de tiempo)

$\mathrm{k}=\left(89^{\circ} \mathrm{C}-36{ }^{\circ} \mathrm{C}\right) / 120 \mathrm{~V}=53 / 120=0.44($ Ganancia $)$

Linealizando la función de transferencia usando la relación de Pade de primer orden:

$$
e^{-L s} \approx \frac{1-\frac{L s}{2}}{1+\frac{L s}{2}}=\frac{-s+\frac{2}{L}}{s+\frac{2}{L}}=\frac{-s+\frac{2}{300}}{s+\frac{2}{300}}=\frac{-s+0.006667}{s+0.006667}
$$

Reemplazando (2) en (1) se obtiene:

$$
G p(s)=\frac{0.44}{750 s+1}\left[e^{-300 s}\right]=\frac{0.44}{750 s+1}\left[\frac{-s+0.006667}{s+0.006667}\right]=\frac{-0.44 s+0.002933}{750 s^{2}+6 s+0.006667}
$$

La función de transferencia, representada por la ecuación (3), es un sistema lineal de segundo orden con dos polos reales $\left(s_{1}=-1 / 750\right.$ y s$\left.=-2 / 300\right)$, siendo el primero el dominante, por lo que se obtiene una respuesta similar a un sistema de primer orden (el polo dominante es 5 veces mayor).

\section{Diseño y simulación del regulador PID para el control de temperatura}

Se ha utilizado el modo óptimo de la herramienta sisotool de Matlab para el diseño del regulador PID, obteniendo los siguientes parámetros: $\mathbf{k}_{\mathbf{p}}=0.02 ; \mathbf{k}_{\mathbf{i}}=0.0015 ; \mathbf{k}_{\mathbf{d}}=0.35$

$\mathrm{K}_{\mathrm{p}}$ : Constante proporcional

$\mathrm{K}_{\mathrm{i}}$ : Constante integral

$\mathrm{K}_{\mathrm{d}}$ : Constante derivativa

El controlador PID se puede expresar, en forma desacoplada, como:

$$
\operatorname{PID}(\mathrm{s})=\mathrm{K}_{\mathrm{p}}+\mathrm{K}_{\mathrm{i}} / \mathrm{s}+\mathrm{K}_{\mathrm{d}} \mathrm{s}=0.02+0.0015 / \mathrm{s}+0.35 \mathrm{~s}
$$

En la Figura 8 se muestra el diagrama de bloques para el control de temperatura PID, utilizando la herramienta SIMULINK.

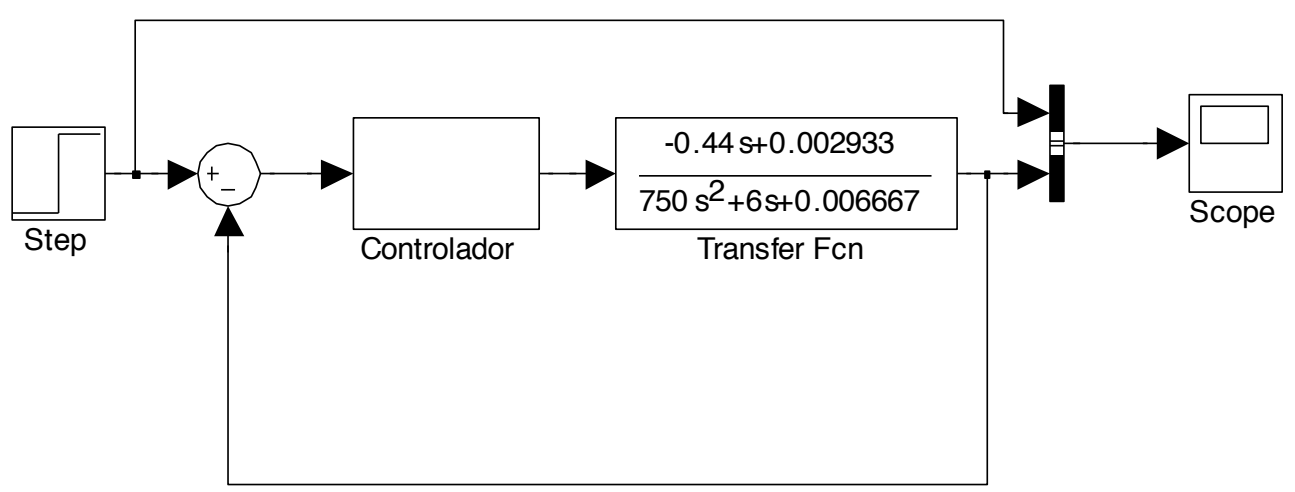

Fig. 8. Diagrama de bloques para el control de temperatura 
En la Figura 9 se muestra la respuesta del sistema de control de temperatura de la Figura 8, donde se observa un tiempo de establecimiento (tiempo en el cual la variable alcanza el set point) de aproximadamente 700 segundos.

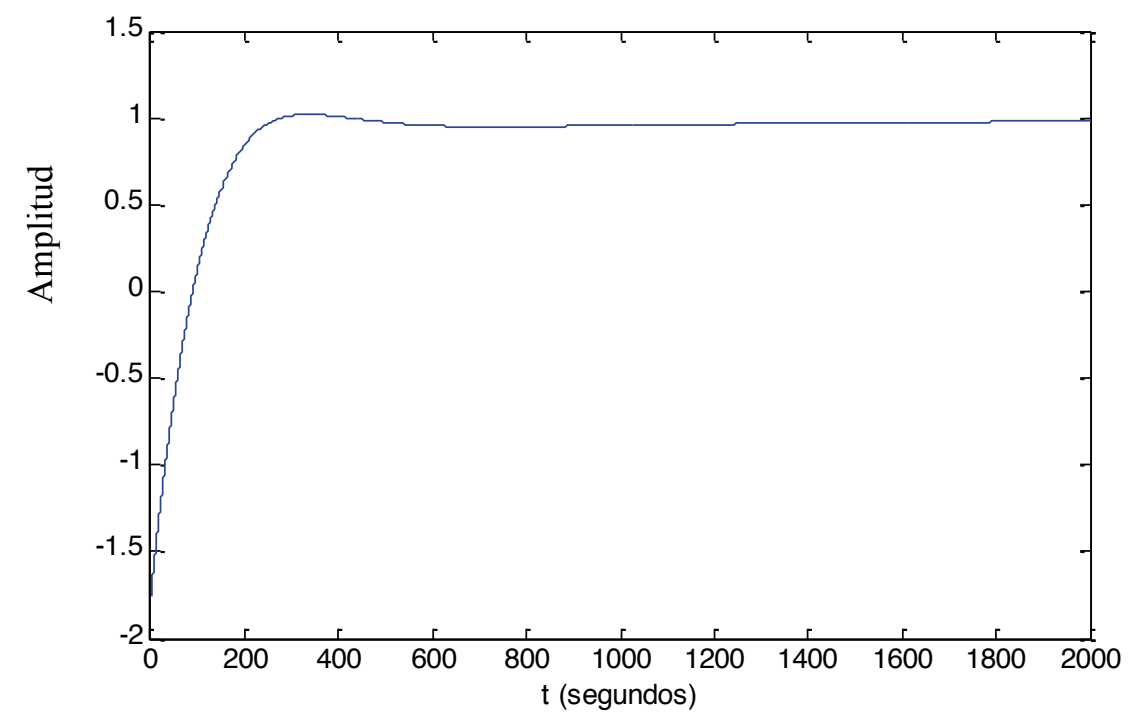

Fig. 9. Respuesta del sistema de simulación del control de temperatura PID.

\section{RESULTADOS EXPERIMENTALES}

En la Figura 10 se observa el diagrama de bloques del sistema en lazo cerrado, para el controlador representado con la ecuación (4) y diseñado en
SIMULINK para el control de temperatura con un regulador PID. Se utilizan los bloques Digital Output y Analog input de la librería RTWINTG (Real Time WINdow TarGet de Matlab) para interactuar físicamente con el módulo didáctico en tiempo real.

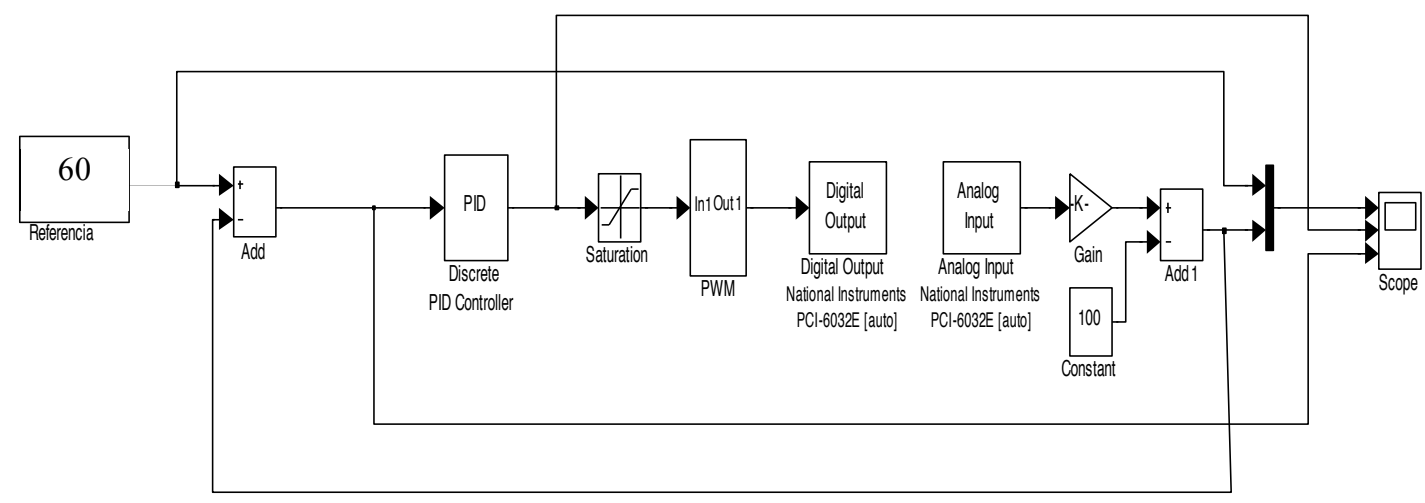

Fig. 10. Diagrama de bloques para el control experimental de temperatura PID

En la Figura 11 se muestra la respuesta del sistema de la Figura 10 para un set-point de $60{ }^{\circ} \mathrm{C}$, un overshoot de 40 ${ }^{\circ} \mathrm{C}$ y un tiempo de establecimiento de $2000 \mathrm{~s}$. 
Estudio del comportamiento de un Control MPC [Control Predictivo Basado en el Modelo] comparado con un Control PID en una Planta de Temperatura

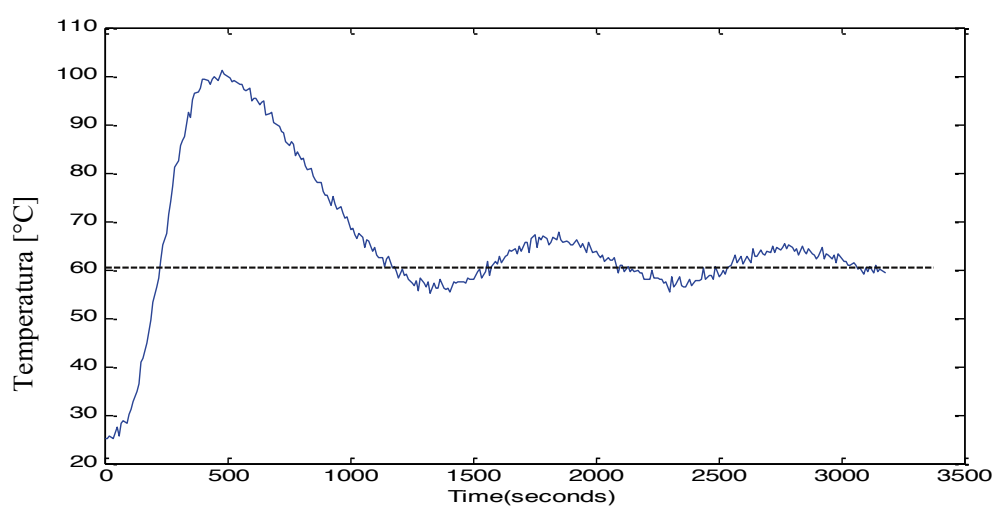

Fig. 11. Respuesta del sistema de control experimental PID de temperatura en lazo cerrado

En la Figura 12 se muestra el diagrama de bloques del sistema en lazo cerrado para el control de temperatura con un regulador MPC.

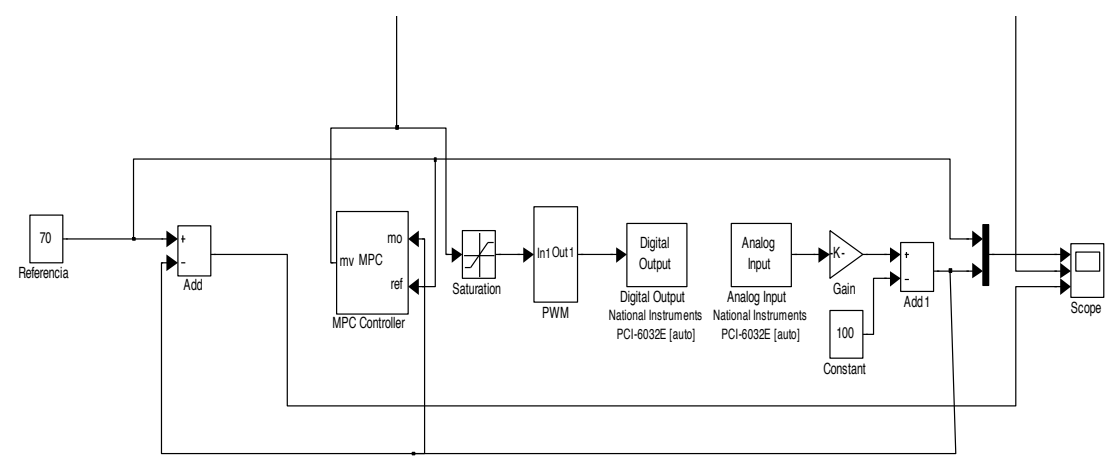

Fig. 12. Diagrama de bloques para el control experimental de temperatura MPC.

En la Figura 13 se muestra la respuesta del sistema de la Figura 12, donde se observa un tiempo de establecimiento de 1000 segundos, evidenciando un mejor comportamiento del Control Predictivo con base en el Modelo (MPC) comparado con el control PID.

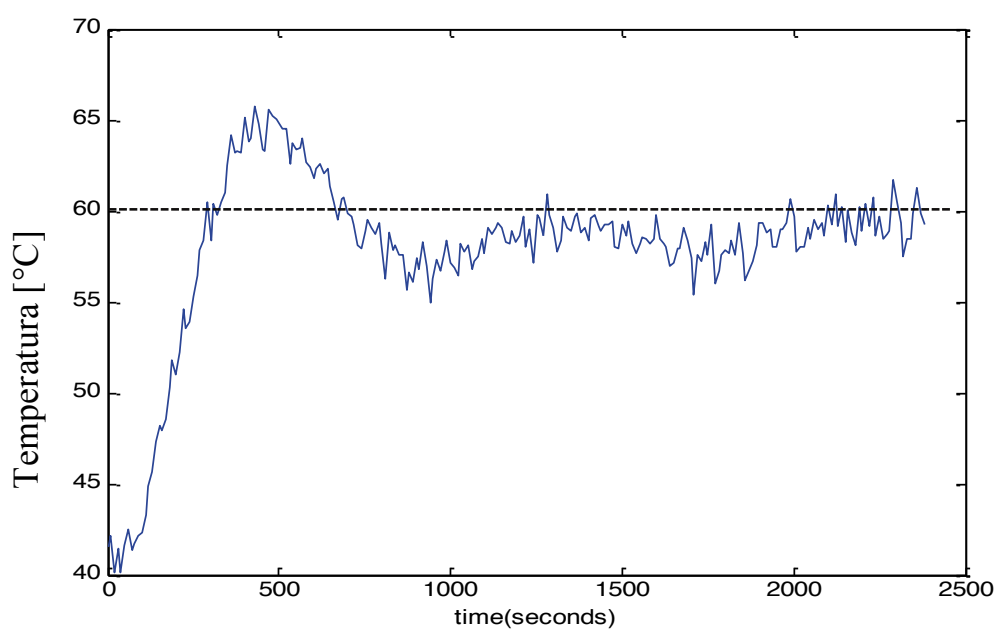

Fig. 13. Respuesta del sistema de control experimental MPC de temperatura en lazo cerrado 
En la Figura 14 se comparan los controladores [MPC y PID] y se observa el mejor comportamiento dinámico del MPC sobre el PID en términos de tiempo de establecimiento y sobreimpulso máximo [12-14]. Los parámetros del control MPC son los siguientes [9]:

Tiempo de muestreo (ts) $=10 \mathrm{~s}$

Horizonte de predicción $\left(\mathrm{H}_{\mathrm{p}}\right)=10$ ts

Horizonte de control $=2$ ts

La Tabla 1 muestra la respuesta transitoria de los dos controladores.

\section{TABLA 1}

RESPUESTA TRANSITORIA DE LOS SISTEMAS DE CONTROL PID Y MPC

\begin{tabular}{|c|c|c|}
\hline Respuesta transitoria & PID & MPC \\
\hline Tiempo de retardo [s] & 125 & 187 \\
\hline Tiempo de subida [s] & 245 & 310 \\
\hline Tiempo pico [s] & 500 & 430 \\
\hline Sobre elongación $\left[{ }^{\circ} \mathrm{C}\right]$ & 40 & 5 \\
\hline Tiempo de asentamiento $[2 \%][\mathrm{s}]$ & 2000 & 1000 \\
\hline
\end{tabular}

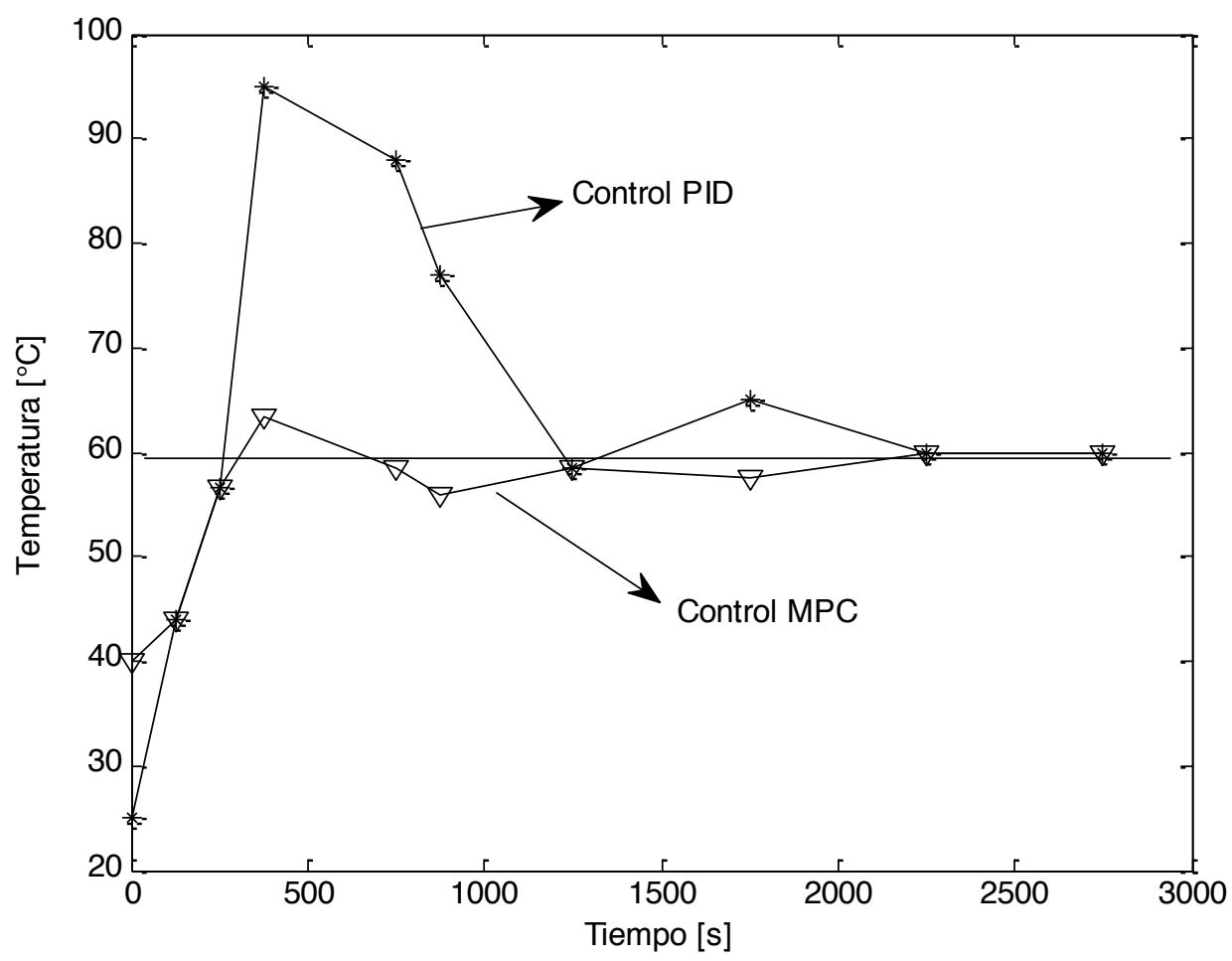

Fig. 14. Respuestas de los sistemas de control PID y MPC

\section{Conclusiones}

Se observa, según los resultados experimentales, que el control MPC tiene mejor comportamiento dinámico, con un tiempo pico menor que el control PID; esto hace posible incrementar el set-point de temperatura más cercano a la restricción por máxima temperatura.
El tiempo de establecimiento del control PID es de 2000 segundos, y del MPC es de 1000 segundos, tomando un $2 \%$ del set point, evidenciando la eficiencia del control predictivo en sistemas con retardo.

La temperatura de sobrepico es una restricción activa. Desde el punto de vista del control de un proceso, el control MPC permanecerá un mayor tiempo en la 
ventana operacional, lo que tiene como consecuencia mayor eficiencia y beneficio económico potencial.

Se ha diseñado e implementado en Matlab un controlador predictivo basado en el modelo (MPC), el cual mejora la respuesta del sistema en lazo cerrado. Para comparar los resultados se elaboró una tabla que evidencia la superioridad del control predictivo en sistemas con retardo.

\section{REFERENCIAS}

[1] K. Ogata, Ingeniería de control moderna. $4 .{ }^{\mathrm{a}} \mathrm{ed}$. España: Pearson Educación, 2003.

[2] R. Salcedo y R. Correa, "Sicompc: Simulador para control predictivo basado en modelos", Dyna, N. ${ }^{\circ} 156$, pp. 89-98, Nov., 2008.

[3] A. Gómez y R. Correa, "Implementación de un sistema de control predictivo multivariable en un horno", Dyna, N. ${ }^{\circ} 157$, pp. 195-203, Marzo-2009.

[4] W. Wittenmark, Computer-Controlled systems: theory and design /. 3rd ed.

[5] K. Astrom, and J. Hagglund, Advanced PID Control. ISA. United States: 2006.
[6] E. Umez, Dinámica de sistemas y control. United States: 2006.

[7] A. Ollero, Control por computadora: Descripción interna y diseño óptimo. México: Alfaomega, 1992, XXIII, 374 p.

[8] R. Cusido, Universidad Politécnica de Cataluña, Control de procesos. 2a.ed. México: Alfaomega, 2002, XIX, $606 \mathrm{p}$

[9] J.M. Maciejowski, Predictive Control with Constraints, Prentice-Hall, Englewood Clifts, NS, 2002.

[10] NI 6013/6014 Family Specifications, (C) 20032005 National Instruments Corporation.

[11] J. Maloney, Electrónica industrial moderna. 5a. edición, México: Pearson Educación, 2006.

[12] PWM Solenoid/Valve Driver, Burr-Brown products from Texas Instruments.

[13] Real-Time Windows Target for Use with RealTime Workshop ${ }^{\circledR}$, User's Guide Version 2. (C) COPYRIGHT 1999-2006 by the MathWorks, Inc.

[14] L. Ljung, T. Glad, Modeling of dynamic systems. 1. ${ }^{\mathrm{a}}$ ed. United States: Prentice Hall, 1994. P. 11. 